\title{
Magnetotunneling as a Probe of Luttinger-Liquid Behavior
}

\author{
Alexander Altland $^{1,2}$, C. H. W. Barnes ${ }^{2}$, F. W. J. Hekking ${ }^{1,2,3}$ and A. J. Schofield ${ }^{2,4}$ \\ 1 Theoretische Physik III, Ruhr-Universität Bochum, 44780 Bochum, Germany \\ 2 Cavendish Laboratory, University of Cambridge, Madingley Road, Cambridge CB3 OHE, United Kingdom \\ ${ }^{3}$ CNRS-CRTBT 8 Université Joseph Fourier, 38042 Grenoble Cedex 9, France \\ 4 School of Physics and Astronomy, University of Birmingham, Birmingham B15 2TT, United Kingdom
}

(September 27, 2018)

\begin{abstract}
A novel method for detecting Luttinger-liquid behavior is proposed. The idea is to measure the tunneling conductance between a quantum wire and a parallel two-dimensional electron system as a function of both the potential difference between them, $V$, and an in-plane magnetic field, $B$. We show that the two-parameter dependence on $B$ and $V$ allows for a determination of the characteristic dependence on wave vector $q$ and frequency $\omega$ of the spectral function, $A_{\mathrm{LL}}(q, \omega)$, of the quantum wire. In particular, the separation of spin and charge in the Luttinger liquid should manifest itself as singularities in the $I$ - $V$-characteristic. The experimental feasibility of the proposal is discussed.
\end{abstract}

PACS numbers: 71.10.Pm, 72.20.-i, 73.40.Gk

The physical properties of a one-dimensional electron system (1DES) are markedly distinct from those of its higher dimensional counterparts: No matter how weak the interactions between particles, the 1DES cannot be described within established Fermi-liquid like pictures of interacting fermions. Rather, it is always unstable towards the formation of a highly correlated state of matter, the so-called Luttinger liquid (LL) [1]. LL-behavior is signalled by the absence of electron-like quasiparticles and instead is characterized by separate low-lying collective excitations associated with spin and charge degrees of freedom. This phenomenon of spin-charge separation and other features identifying LL phases have been studied extensively and various excellent reviews on the subject exist [2 [6]. The continued research activity on onedimensional systems is not merely of academic interest as there are a growing number of physical applications: organic polymers [7]; carbon nanotubes [8]; quantum Hall edge states [9,10]; and ultra-narrow quantum wires [1] are believed to fall into the general class of 1DES's.

Despite this the present experimental situation is inconclusive. Although previous studies on organic conductors and superconductors, inorganic charge density wave materials, semiconductor quantum wires and fractional quantum Hall phases (see [5] for a more extensive list of references) have been consistent with various aspects of the highly correlated behavior of 1DES's, an unambiguous experimental observation of a LL-phase is still lacking.

In this Letter we propose a novel experimentfalling into the general class of semiconductor transport measurements - which should provide evidence for spincharge separation in 1D. The basic experimental device is displayed in Fig. 1. A 1DES runs at a height $d$ above a parallel two-dimensional electron system (2DES). The 1DES and the 2DES are kept at a relative voltage $V$ and an in-plane magnetic field is applied with a component $B$ perpendicular to the wire. A setup of this type may be realized in a number of ways: a double quantum well (DQW) heterostructure patterned with appropriate external gates [12]; a suitably etched resonant tunneling diode [13]; or an organic polymer or carbon nanotube with an electrical contact at one end [7,8] placed on an undoped heterostructure with a shallow 2DES. The presence of a voltage bias induces the flow of a tunnel current $I(V, B)$ between the 1 DES and the 2DES. As will be detailed below, $I(V, B)$ is essentially determined by the overlap of the spectral functions $A_{i},(i=2 D, 1 D)$ of the two subsystems. By fine-tuning the control parameters $V$ and $B$ the overlap integral changes in a pronounced way, thereby probing features of both $A_{1 D}$ and the (essentially known) $A_{2 D}$. The former is believed to be governed by the phenomenon of spin-charge separation. In this way, the 2DES can be employed as a 'spectrometer' scanning the LL characteristics of the quantum wire.

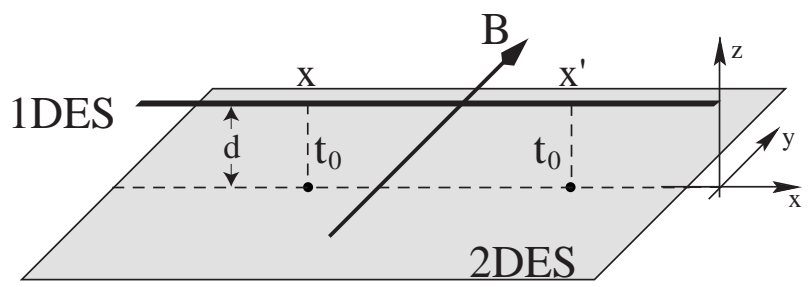

FIG. 1. Device configuration for magnetotunneling between a 1 DES and a 2 DES.

To formulate the above program quantitatively we model the device depicted in Fig. 1 in terms of the Hamiltonian

$$
H=H_{1 D}+H_{2 D}+H_{T},
$$

where $H_{1 D}, H_{2 D}$ describe the $1 \mathrm{DES}$ and the 2DES, respectively. The tunnel Hamiltonian $H_{T}$ transfers elec- 
trons between the 1DES and the 2DES. It is modeled as

$$
H_{T}=t_{0} \int d x\left[e^{-i e d B x} \Psi_{2 D, s}^{\dagger}(x) \Psi_{1 D, s}(x)+\text { h.c. }\right],
$$

where $\Psi_{i, s}, i=1 D, 2 D$ are fermionic field operators with spin $s=\uparrow, \downarrow$ and $\Psi_{2 D}(x)$ is a shorthand for the 2DES field operator evaluated at point $\mathbf{x}=(x, 0)$ (see Fig. 1). We have chosen a gauge where the entire dependence on the magnetic field is contained in the Aharonov-Bohm phases carried by the matrix elements of $H_{T}$. [In passing we note that the magnetic field needed to drive the effects discussed below is weak: $B \sim \mathrm{eV} /\left(d v_{F}\right)$, where $v_{F}$ is the Fermi velocity of the 2DES. Fields of this type are not expected to affect the bulk physics of both the 1DES and the 2DES.] In writing (11) and (2) two essential approximations have been made: First, drag effects (i.e. electron-electron interactions between 1DES and 2DES) are neglected. The justification is that at the low temperatures considered here, standard Fermi liquid arguments applied to the 2DES show that drag effects are suppressed by a phase space factor $\sim T^{2}$ at temperature $T$. Second, it is assumed that tunneling occurs between neighboring points $x \in 1$ DES $\leftrightarrow \mathbf{x} \in$ 2DES only (with amplitude $t_{0}$ ). Owing to the exponential dependence of the tunneling amplitude on both the height of the tunneling barrier and the tunneling distance, direct processes are the most relevant by far. By virtue of this assumption, the problem becomes effectively one-dimensional.

To leading order in the amplitude $t_{0}$ the tunnel current per unit length is given by [14]:

$$
\begin{gathered}
I(V, B)=\frac{4 I_{0}}{m} \int d q \int \frac{d \epsilon}{2 \pi}[f(\epsilon-e V)-f(\epsilon)] \\
\times A_{1 D}(q, \epsilon) A_{2 D}\left(q-q_{B}, \epsilon-e V\right),
\end{gathered}
$$

where $f(\epsilon)$ is the Fermi function, $m$ the 2DES electron mass and $I_{0}=e\left|t_{0}\right|^{2} m / \pi$ the natural unit of current in the problem. The spectral functions $A_{i}(q, \omega)=$ $-2 \Im \mathrm{m} G_{i}^{R}(q, \omega)$, where $G_{i}^{R}(q, \omega)$ are the Fourier transforms of the retarded Green functions $G_{i}^{R}(x, t)=$ $-i \theta(t)\left\langle\left\{\Psi_{i, s}(x, t), \Psi_{i, s}^{\dagger}(0,0)\right\}\right\rangle[15]$.

The structure of the above integral representation of $I(V, B)$ already reveals the basic idea of this Letter: according to (3) the current is given by the overlap of the two spectral functions integrated over a window of width $\max (T, e V)$ at the Fermi energy. As detailed below, the value of the overlap integral sensitively depends on the two parameters $e V$ and $q_{B} \equiv e B d+k_{F}^{2 D}-k_{F}^{1 D}$ which shift the relative origin of the two spectral functions. $k_{F}^{1 D / 2 D}$ are the Fermi wave vectors of the 1DES and 2DES respectively. The 1DES $A_{1 D}(q, \omega)$ is expected to exhibit pronounced structures depending in a non-trivial way on LL characteristics, whereas the spectral function of the 2DES is dominated by electron-like quasiparticles and its important features are explicitly known. Thus, $A_{2 D}$ may serve as a 'spectrometer' scanning the features of $A_{1 D}$ as $q_{B}$ and $\mathrm{eV}$ are varied. In particular, assuming that $A_{1 D}$ is of LL type we show below that the tunnel current is profoundly affected by the phenomenon of spin-charge separation which should give a clear signal of LL behavior.
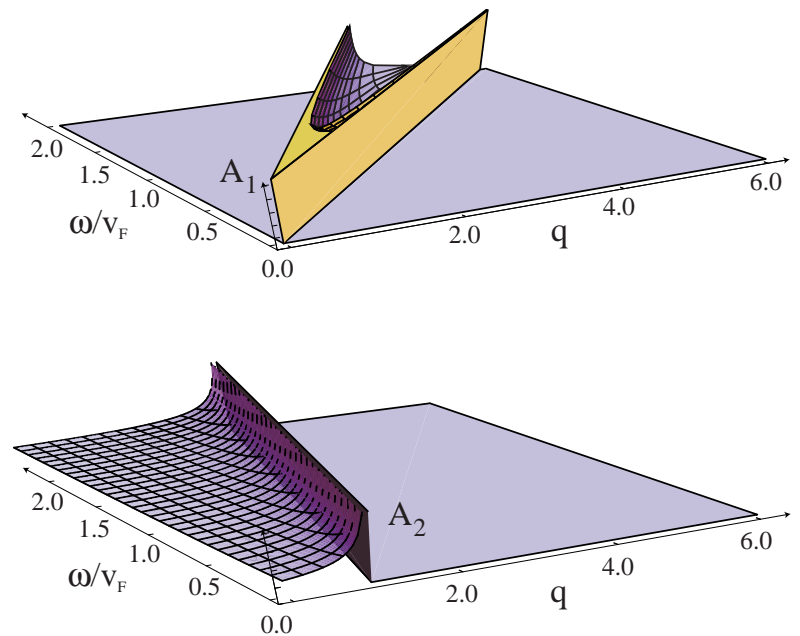

FIG. 2. Plot of the two spectral functions in the $(q, \omega)$ plane (arbitrary units) for $v_{\rho}=v_{F} / 2, v_{\sigma}=v_{F} / 3$ and $r=1.5$.

We proceed by specifying the spectral functions employed in calculating the current. Owing to the onedimensionality of the problem both functions $A_{i}$ can be decomposed according to $A_{i}(q)=\sum_{\eta= \pm 1} A_{i, \eta}\left(q_{\eta}, \omega\right)$, where $A_{i, 1}\left(A_{i,-1}\right)$ represents the contribution of rightand left-moving charge carriers, respectively. Assuming that both interactions and disorder are negligible (an assumption we discuss below), the function $A_{2 D}$ in the vicinity of the Fermi surface is then given by (see Fig. 2)

$$
A_{2 D, \eta}(q, \omega)=\sqrt{2 m} \frac{\Theta\left(\omega-\eta q v_{F}\right)}{\sqrt{\omega-\eta q v_{F}}} .
$$

As for $A_{1 D}$, various forms of LL spectral functions have been discussed in the literature. We here employ the function (see Fig. 2)

$$
\begin{aligned}
& A_{1 D, \eta}(q, \epsilon)=2 \\
& \frac{\Theta\left(\epsilon-\eta q v_{\sigma}\right) \Theta\left(\eta q v_{\rho}-\epsilon\right)+\Theta\left(\eta q v_{\sigma}-\epsilon\right) \Theta\left(\epsilon-\eta q v_{\rho}\right)}{\sqrt{\left|\epsilon-\eta q v_{\rho}\right|\left|\epsilon-\eta q v_{\sigma}\right|}},
\end{aligned}
$$

where $v_{\sigma}$ and $v_{\rho}$ are the velocities of spin and charge density waves, respectively. For the type of systems considered here, $v_{F}>v_{\rho}>v_{\sigma}$ [16]. Eq.(5) was derived in Ref. [5] under the simplifying assumption of no interactions between left and right moving particles (formally that the Luttinger-Liquid parameter $K_{\rho}=1$ ). This condition can be relaxed at the expense of the appearance of spectral weight outside the limits defined by (5). 
This does not alter the main conclusions of this Letter but would add considerably to the complexity of exposition. We therefore leave it for subsequent discussion [17]. Substituting Eqs. (4) and (5) into (3), we find that four regimes $R_{j}, j=1, \ldots, 4$ with qualitatively different behavior exist. Introducing dimensionless parameters $r=1+q_{B} v_{F} / e V, a_{\rho}=v_{F} / v_{\rho}$ and $a_{\sigma}=v_{F} / v_{\sigma}$, these are given by $R_{1}: r<1, R_{2}: 1 \leq r \leq a_{\rho}, R_{3}: a_{\rho} \leq$ $r<a_{\sigma}$ and $R_{4}: r>a_{\sigma}$. A schematic plot of the relative positioning of the spectral functions in the regimes $R_{1}, \ldots, R_{4}$, respectively, is shown in Fig. 3 as a function of the dimensionless 1DES wave vector $x=q v_{F} / e V$ and frequency $s=\omega / \mathrm{eV}$.
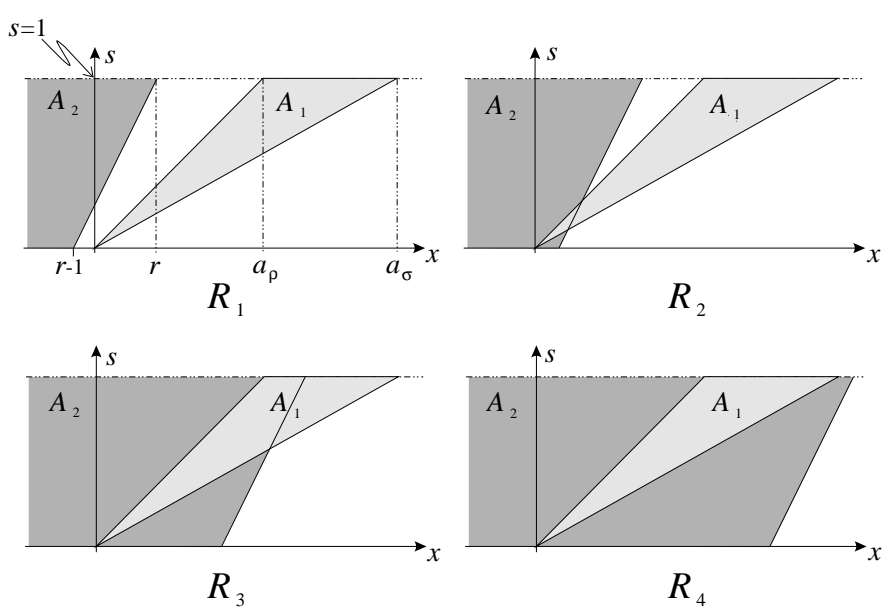

FIG. 3. Relative position of the two spectral functions in the four regimes $R_{1}, \ldots, R_{4}$. The light (dark) shaded areas represent the functions $A_{1 D}\left(A_{2 D}\right)$.

The current can now be obtained by double integration over $q$ and $\omega$. In all regimes the integrations can be carried out in closed form although the resulting formulae tend to be somewhat lengthy and partly involve special functions so will be discussed elsewhere [17]. Here we restrict ourselves to a discussion of the current in the asymptotic regions where adjacent regimes meet (and the sensitivity of the result to variations in the external parameters is most pronounced).

Figure 1 shows both $I$ and the differential conductance $G \equiv d I / d V$ at $T=0$ plotted as a function of the parameter $r$. We note here that variation of $r$ may be achieved not only by varying $B$ but also by changing the relative 2DES or 1DES carrier densities. However, this would introduce the possibility for capacitive coupling effects which would make the determination of the LL parameters more difficult 17]. In the following we discuss the behavior of the result in the various regimes separately.

$R_{1}$ : The two spectral functions $A_{1 D}$ and $A_{2 D}$ do not overlap (cf. Fig. 3) implying that the current vanishes.

$R_{2}$ : For $r>1$, the spectral functions start to overlap leading to a (singular) onset of current flow. At the same time the conductance diverges as $\tilde{g} \sim-(r-1)^{-1 / 2}$, where we have introduced $\left.\tilde{g} \equiv G \sqrt{V e^{-1} E_{F}} / I_{0}\right)$ as a dimensionless measure for the conductance. The inverse square root behavior of the conductance persists up to the boundary to $R_{3}$ where $g\left(r \rightarrow a_{\rho}\right)=-\left(a_{\rho}-\right.$ $1)^{-1}\left(a_{\rho} a_{\sigma}\left(a_{\sigma}-1\right)\right)^{1 / 2}$.

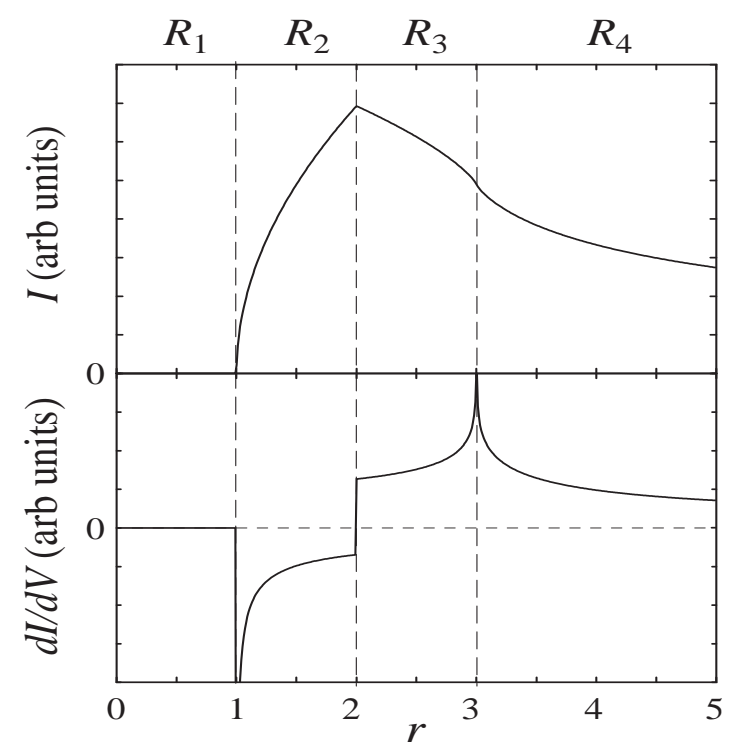

FIG. 4. Tunneling current and differential conductance as a function of the magnetic field $\left(a_{\rho}=2, a_{\sigma}=3\right)$.

$R_{3}$ : As $r$ crosses over into $R_{3}$, the conductance exhibits a second discontinuity, the magnitude of which is found to be

$$
g\left(a_{\rho}^{+}\right)-g\left(a_{\rho}^{-}\right)=\frac{a_{\rho}}{a_{\rho}-1} \sqrt{\frac{a_{\rho} a_{\sigma}}{a_{\sigma}-a_{\rho}}},
$$

where $a_{\rho}^{ \pm}=a_{\rho} \pm \delta, \delta$ infinitesimal. Note that the jump is accompanied by a change of sign. As $r$ approaches the boundary to $R_{4}$, the conductance again exhibits a singularity, this time of logarithmic type. More precisely,

$$
g(r) \stackrel{r \rightarrow a_{\sigma}}{\longrightarrow}-\frac{a_{\sigma}}{a_{\sigma}-1} \sqrt{\frac{a_{\rho} a_{\sigma}}{a_{\sigma}-a_{\rho}}} \frac{1}{\pi} \ln \left(a_{\sigma}-r\right) .
$$

$R_{4}$ : The boundary singularity at $a_{\rho}$ turns out to be symmetric, i.e. for small $\epsilon, g\left(r=a^{\sigma}+\epsilon\right)=g\left(r=a^{\sigma}-\epsilon\right)$. Eventually, for asymptotically large $r$ the conductance decays as $g \sim r^{-1 / 2}$.

In summary we see that the structure of the $I-V$ characteristic is essentially determined by the two spin-charge parameters $a_{\rho}$ and $a_{\sigma}$. For a more general Luttingerliquid, with $K_{\rho} \neq 1$, the power laws associated with the singular features will be modified but their location is determined by $a_{\rho}$ and $a_{\sigma}$ allowing these parameters to be measured. In order to decide whether this strategy of demonstrating LL behavior is practical it is imperative to estimate the effect of two ingredients that tend to blur the above sharp structures of the $I-V$-curve: finite temperatures and disorder. 
As for the effect of finite temperatures, it is intuitively clear that the structures of the $I-V$ characteristics will be completely smeared for $T$ larger than any of the characteristic energy scales $\left(e V a_{\rho, \sigma}, q_{B} v_{F} a_{\rho, \sigma}\right.$ or any combination thereof) of the problem. (To see this more explicitly, notice that for finite $T$ the integration in (3) no longer extends over a sharply defined strip in the $(q, \omega)$ plane but rather over a smeared region of width $\mathrm{eV} \pm T$ ). However, it has been demonstrated for 2D-2D and 1D$2 \mathrm{D}$ tunneling in DQW structures that at temperatures readily available in experiment its effect may be ignored 12, 18, 19 .

The effects of disorder are more significant and can be considered individually for the 2DES and 1DES. For the 2DES, 2D-2D tunneling measurements show an effective blurring of $A_{2 D}$ over an energy range $\Gamma$, where $\tau=\Gamma^{-1}$ is the average scattering time in the 2DES. Optimizing $\Gamma$ to be smaller than the characteristic energy scales of the problem (see above) is therefore necessary for the observability of the above effects. In the best GaAs/AlGaAs DQW systems $\Gamma \sim 0.25 \mathrm{meV}$ [18] and the condition $e V>\Gamma$ can be easily satisfied. 19].

As for the 1DES, the effects of disorder should be largely absent in the consideration of carbon nanotubes and organic polymers themselves. However achieving a 2DES sufficiently close to a heterostructure surface to allow tunneling into these systems is a technologically difficult problem and the resulting 2DES is likely to have a larger $\Gamma$ than a fully optimized DQW structure [20]. For a surface gate defined 1DES in a DQW the remote ionized impurities, random impurities and crystal faults could be strong enough to pin its low lying excitations thereby destroying the LL behavior. However, provided this does not happen, i.e. assuming that a LL phase in quantum wires may exist in principle [21], we expect the disorder to effectively renormalize the characteristic LL parameters, most notably the spin and charge density wave velocities. Similarly, variations in the thickness of the tunnel barrier can in principle have a large effect on tunneling rates and therefore the clarity of any measured signal. At any rate, neither the presence of remote impurities nor tunnel barrier variations have prevented experiments in high mobility DQW systems from clearly resolving structures of the spectral functions of quasione-dimensional systems [12].

Summarizing, we have proposed an experiment which should allow the detection of Luttinger liquid behavior in a 1DES by detecting magnetotunneling between the 1DES and a parallel 2DES. We have shown how the parameters characterizing a LL, the ratio of spin and charge velocity, can be determined from the voltage and/or magnetic field dependence of the tunneling conductance. It was argued that, notwithstanding the presence of thermal and disorder smearing effects, the experiment should be feasible by means of today's technology.

Acknowledgements: We are grateful to I. Aleiner and
C. J. B Ford for instructive discussions. FWJH acknowledges the financial support of the European Community (contract ERB-CHBI-CT941764); AA and FWJH acknowledge the support of the Deutsche Forschungsgemeinschaft through SFB 237. CHWB and AJS acknowledge the support of the EPSRC and the Royal Society respectively.

[1] F. D. M. Haldane, J. Phys. C, 14, 2585 (1981).

[2] V. J. Emery, in Highly Conducting One-Dimensional Solids, edited by J. T. Devreese, R. P. Evrard, and V. E. van Doren (Plenum, New York, 1979).

[3] J. Sólyom, Adv. Phys. 28, 201 (1979).

[4] H. J. Schulz, in Mesoscopic Quantum Physics, edited by E. Akkermans, G. Montambaux, J. -L. Pichard, and J. Zinn-Justin (North-Holland, Amsterdam, 1995).

[5] J. Voit, Phys. Rev. B 47, 6740 (1993); Rep. Prog. Phys., 58, 977 (1995). H.J. Schulz et al., cond-mat/9807366.

[6] M.P.A. Fisher and L.I. Glazman in Mesoscopic Electron Transport, edited by L. Kouwenhoven, G. Schön, and L. Sohn (Kluwer Ac. Publ., Dordrecht, 1997).

[7] A.J. Heeger et al Rev. Mod. Phys. 60, 781 (1988).

[8] A. Thess et al., Science 273, 483 (1996).

[9] X.G. Wen, Phys. Rev. B 41, 12838 (1990).

[10] F.P. Milliken, C.P. Umbach, and R.A. Webb, Solid State Commun. 97, 309 (1996).

[11] S. Tarucha et al., Sol. State Comm. 94, 413 (1995); A. Yacoby et al., Phys. Rev. Lett. 76, 4612 (1996).

[12] B. Kardynał et al., Phys. Rev. B 55, R1966 (1997)

[13] P. H. Beton et al., Phys. Rev. Lett. 75, 1996 (1995).

[14] See, e.g., G.D. Mahan, Many-Particle Physics (Plenum, New York, 1990).

[15] $A_{2 D}$ is defined as the $1 D$ Fourier transform of the $2 D$ spectral function evaluated on the line $(x, 0)$ and involves an integration over all momenta in $y$-direction. As a consequence $A_{2 D}(q, \omega)$ contains spectral weight for small momenta $\left|v_{F} q\right|<\omega$. The physical meaning of this 'shadow region' is that states propagating between two points on the line $(x, 0)$ can 'detour' through the extended plane, thereby propagating effectively slower than with the Fermi velocity. We use a non-interacting form to caption the singular structure of $A_{2 D}$ due to quasiparticles at $T=0$.

[16] For repulsive interactions, $v_{\rho}>v_{\sigma}$ holds in general. The first inequality $v_{F}>v_{\rho}$ is a condition which can be set experimentally by varying the 2DES density.

[17] A. Altland, C.H.W. Barnes, F.W.J. Hekking and A. J. Schofield, to be published.

[18] S. Q. Murphy et al., Phys. Rev. B 52, 14825 (1995).

[19] N. Turner et al., Phys. Rev. B 54, 10614 (1996).

[20] D.A. Ritchie private communication.

[21] In any given realization of this experiment it would be possible to determine whether disorder in the $1 \mathrm{D}$ system is disrupting the Luttinger liquid state by monitoring the conductance of the wire and ensuring that it is $2 e^{2} / h$. 